\title{
Glioblastoma Multiforme with Hemorrhage Mimicking an Aneurysm: Lessons Learnt
}

\author{
Navneet Singla ${ }^{a} \quad$ Ashish Aggarwala $^{a}$ Sameer Vyas ${ }^{b} \quad$ Ankur Sanghvi $^{a}$ \\ Pravin Salunke ${ }^{a}$ Ravi Garg ${ }^{a}$ \\ Departments of a Neurosurgery and ${ }^{b}$ Radiodiagnosis, Post Graduate Institute of Medical Education and Research, \\ Chandigarh, India
}

\section{Key Words}

Aneurysm · Glioblastoma · Intratumoral hemorrhage

\begin{abstract}
Background: A sudden onset of neurological symptoms in patients is conventionally thought to be due to vascular phenomenon, with one common differential diagnosis being subarachnoid hemorrhage. Another important differential diagnosis is ischemic stroke. An uncommon cause of such acute symptoms can be hemorrhage in a pre-existing tumor, that is, intratumoral hemorrhage (ITH). Purpose: ITH is an important, though uncommon differential diagnosis in cases of sudden onset of neurological deterioration. Methods and Result: A 60-year-old male presented with seizures and loss of consciousness $12 \mathrm{~h}$ prior to admission. The episode was sudden in onset. After detailed clinical and radiological investigations, the patient was diagnosed with glioma with bleed and was successfully operated upon. Conclusion: The combination of hemorrhage and ischemic stroke pointed more towards an aneurysm rather than a tumor bleed. There were pointers both in favor of and against both the diagnosis. Therefore, a complex hemorrhagic cerebral tumor with acute presentation and discordant finding on CT or CT angiography should be characterized preoperatively. A large thrombosed aneurysm remains an important differential diagnosis.

(c) 2016 S. Karger AG, Basel
\end{abstract}

\section{Introduction}

Patients presenting with sudden onset neurological symptoms, conventionally thought to be due to vascular phenomenon, have one common differential diagnosis subarachnoid hemorrhage. Another important differential is ischemic stroke. An uncommon cause of such acute symptoms can be hemorrhage in a pre-existing tumor, that is, intratumoral hemorrhage [1]. In this case, suddenness of symptoms, morphology of lesion, closeness to a major intracranial vessel, presence of infarct - all lead us to diagnose tumor with bleed with a differential diagnosis of ruptured large aneurysm.

\section{Case Report}

A 60-year-old male presented with seizures and loss of consciousness $12 \mathrm{~h}$ prior to admission. The episode was sudden in onset. On examination, the patient was aphasic with right hemiparesis with no papilledema. Patient was a known hypertensive and chronic smoker. Non-contrast CT (NCCT) head (fig. 1a) showed a circumscribed lesion in the left anterior medial temporal lobe, which was heterogeneously hypodense with hyperdense areas posteriorly suggesting an acute hemorrhage. There was mild perilesional edema. A provisional diagnosis of large partially/completely thrombosed aneurysm (with recent bleed) arising from the left middle cerebral artery (MCA) was made. Cerebral CT angiography (CTA; fig. 1b) revealed non-opacification of left MCA. Furthermore, a dig-

\section{KARGER}

E-Mail karger@karger.com

www.karger.com/aon (c) 2016 S. Karger AG, Basel

0972-7531/16/0234-0263\$39.50/0
Ashish Aggarwal

Department of Neurosurgery

PGIMER, Chandigarh 160012 (India)

E-Mail aaggarwal_7@yahoo.com 

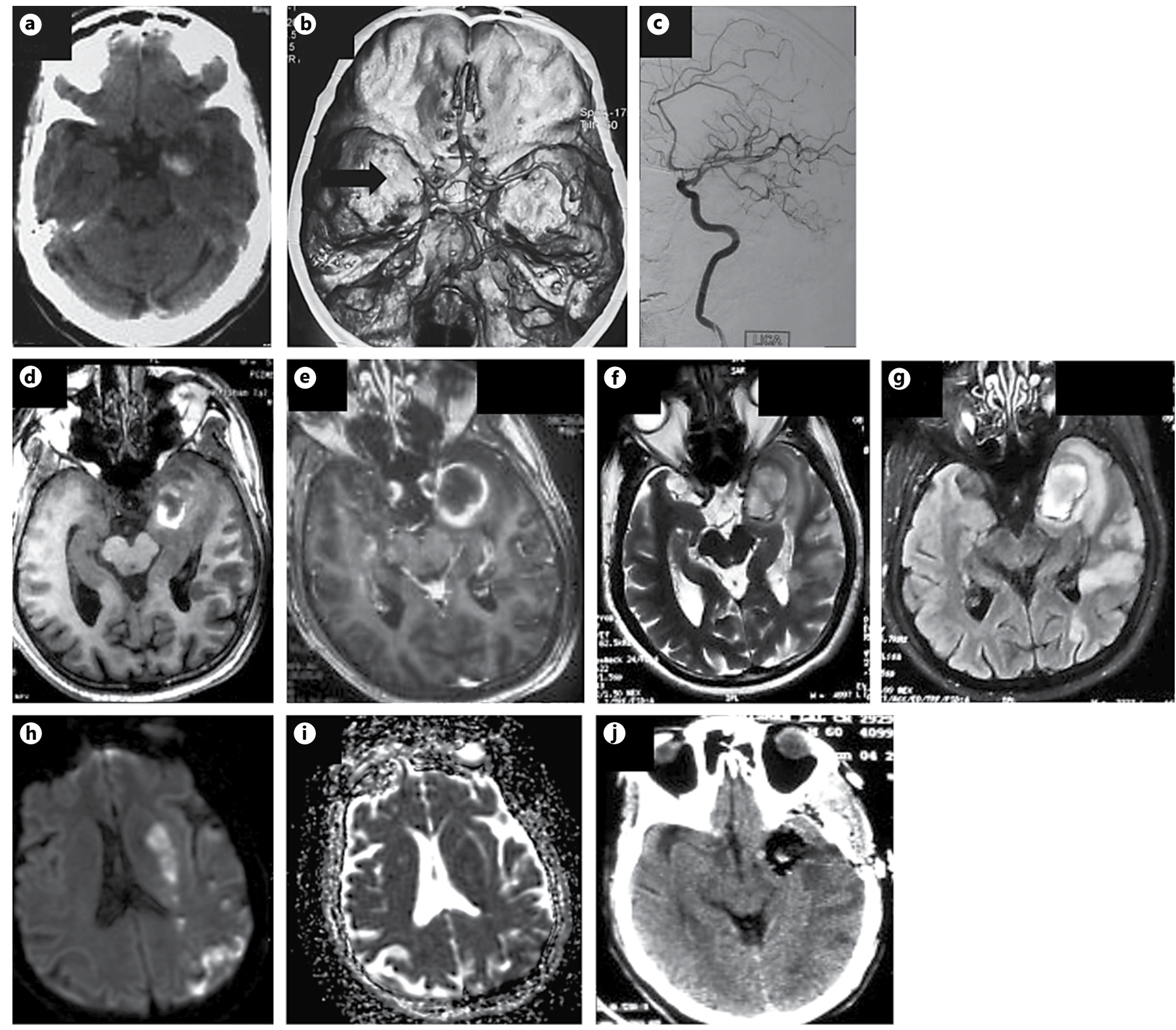

Fig. 1. NCCT head (a) showing a hypodense lesion in left temporal lobe with hemorrhage. CTA (b) revealing non-opacification of left MCA (shown by arrow). DSA (c) showing extrinsic compression of left MCA with no evidence of aneurysm. MRI T1WI plain and contrast $(\mathbf{d}, \mathbf{e})$, shows heterogeneous hemorrhagic lesion with mild

perilesional edema and thick irregular peripheral enhancement. T2 (f) and FLAIR (g) images showing hyperintense lesion with rim of hypointensity. DWI (h) and ADC (i) images showing acute infarct in left basal ganglia. $\mathbf{j}$ Post operative NCCT showing gross total excision.

ital subtraction angiography (DSA; fig. 1c) was done, which revealed extrinsic compression of left MCA with no evidence of aneurysm. At this stage, the diagnosis was again reviewed and a possibility of tumor with bleed was considered. Contrast enhanced MRI (fig. 1d-g) revealed a circumscribed heterogeneous hemorrhagic lesion with mild perilesional edema in left medial temporal lobe. The lesion was hyperintense on T2-weighted images and showed subacute hemorrhage in the posterior aspect. There was thick irregular peripheral enhancement of the lesion. The lesion

was found to abutting the MCA. Diffusion weighted image (DWI) and apparent diffusion coefficient (ADC) images (fig. 1h, i) showed acute infarct in left basal ganglia and left fronto-parietal lobes.

With a diagnosis of high-grade glioma, the patient was taken up for surgery. Left temporal craniotomy and transcortical route through inferior temporal gyrus was taken. There were areas of bleed present. The tumor was soft, suckable with central areas of necrosis. It was abutting MCA and its branches. Gross total excision was achieved (fig. 1j). Histopathology was glioblastoma multiforme. 


\section{Discussion}

In this case, we were initially misled to a diagnosis of thrombosed aneurysm because of - (a) history suggestive of ictus (i.e. sudden deterioration), (b) right hemiparesis, (c) circumscribed hemorrhagic lesion in left temporal region very close to MCA, and (d) no/minimal mass effect. Further cerebral CTA and DSA showed non-opacification of MCA, which was attributed to thrombo-embolism from a large thrombosed aneurysm with complete occlusion of neck.

MRI revealed that the lesion was hemorrhagic with blood in different stages of evolution, incomplete thick hemosiderin rim and irregular enhancement of tumor wall suggestive of high-grade glioma [2]. However, even on MRI some findings remained inconsistent with a diagnosis of high-grade glioma with bleed. These unexplained findings include: (a) minimal mass effect [2], (b) extrinsic compression causing complete, sudden onset cut-off of MCA on CTA which is uncommon in glioma [3], and (c) acute infarct in the region of left basal ganglia. The last finding continued to perplex us, and plausible explanations of bleed in tumor causing vasospasm, tumor embolism was considered.
The combination of hemorrhage and ischemic stroke misled us to think of an aneurysm rather than a tumor bleed. There were pointers both in favor of as well as against the diagnosis (vide supra). Therefore, a complex hemorrhagic cerebral tumor with acute presentation and discordant finding on CT or CTA should be characterized preoperatively. A large thrombosed aneurysm remains an important differential diagnosis.

\section{Author Contribution}

A.A. contributed to the conception and design, interpretation, draft of manuscript and gave the final approval. N.S. contributed to design, data acquisition and gave the final approval. A.S., S.V. and R.G. contributed to design, data acquisition, critically revised manuscript and gave the final approval. P.S. contributed to conception, data analysis, critically revised manuscript and gave the final approval.

\section{Disclosure Statements}

The authors declare no conflict of interest. The article complies with the International Committee of Medical Journal Editor's guidelines.

\section{References}

1 Aggarwal A, Vaiphei K, Savardekar AR, Salunke P, Mathuriya SN: Stroke in three patients due to spontaneous intra tumoral hemorrhage in meningiomas: lessons learnt. J Neurosci Rural Pract 2016;7:183-186.
2 Ideguchi M, Kajiwara K, Goto H, Sugimoto K, Nomura S, Ikeda E, Suzuki M: MRI findings and pathological features in early-stage glioblastoma. J Neurooncol 2015;123:289-297.
3 Kishikawa $\mathrm{H}$, Iwatsuki $\mathrm{K}$, Umeda A: Middle cerebral arterial occlusion secondary to brain tumor - case report. No Shinkei Geka 1979;7: $377-382$. 